\title{
Study of PFS and PAC coagulation effect on Pi River water
}

\author{
Wan-fen Wang ${ }^{1, *}$ \\ ${ }^{1}$ College of Architecture and Civil Engineering, West AnHui University Lu'an 237012
}

\begin{abstract}
Using poly-aluminum chloride (PAC) and poly-ferric sulfate (PFS) as coagulants, the effects of the dosage of PAC and PFS on the removal of turbidity, chromaticity and $\mathrm{UV}_{254^{*}}$ in water were investigated by using the method of enhanced coagulation. The results show that when the raw water is neutral and the turbidity is less than $20 \mathrm{NTU}$, and the $\mathrm{pH}$ value does not decrease much after coagulation and sedimentation, PAC and PFS are separately added to $7.5 \mathrm{mg} / 1,35 \mathrm{mg} / 1$. The removal rate of turbidity and chromaticity in the water sample can reach $83.75 \%$ and $48.47 \%$, respectively, but the removal rate of $U_{2} V_{24}$ is less than $30 \%$, the mass ratio of PAC to PFS is $1: 1$ to $6: 1$, the removal efficiency of turbidity and Chroma increased by about $10 \%$, but the removal efficiency of $\mathrm{UV}_{254 *}$ did not change or even decreased, both PFS and PAC can effectively remove the turbidity and Chroma in the micro polluted Pi River, but the removal effect of $U^{254^{*}}$ is not good, and the removal effect of PAC is better than PFS.
\end{abstract}

\section{Introduction}

he Pi River main canal is a national strategic high-quality water source and also an important drinking water source in Lu'an and Hefei, which is related to the drinking water safety of more than 5 million people in the two cities. The current water quality is basically between class II and III. However, with the acceleration of urbanization construction and the rise of the central economy, in recent years the water quality has shown slow downward trend in some river sections ${ }^{[1]}$.At present, most of the water supply plants in Pi River basin generally use PAC coagulant, which has good removal effect on turbidity and Chroma, but the removal effect of DOM is not ideal.Compared with the low toxicity of aluminum flocculant, iron flocculant has many advantages such as Polymerized ferric sulfate (PFS), which is widely used in the field of water treatment due to its advantages of short coagulation reaction time, low price, wide range of $\mathrm{pH}$ application, less residual iron ions and good dehydration performance of hydrolysate ${ }^{[2]}$.

Enhanced coagulation is widely used in water treatment because of its simple process transformation, low cost, strong operability and obvious effect. The concrete ways of using enhanced coagulation process to treat low turbidity water mainly include reasonable selection of coagulation several methods, such as coagulant and coagulant aid ${ }^{[3]}$, chemical preoxidation, sludge reflux. In this study, the effects of PAC and PFS mass ratio, dosage, raw water turbidity on turbidity reduction, decolorization and organic matter removal of $\mathrm{Pi}$ River water were investigated. The results provide a feasible way for $\mathrm{Pi}$ River Basin water production enterprises to cope with the continuous improvement of water quality, and lay a foundation for the study of coagulation mechanism of PAC and PFS.

\section{Materials and methods}

\subsection{Raw water quality}

The water quality index are that temperature is $15-30{ }^{\circ} \mathrm{C}$, Chroma is $9.8-26.5, \mathrm{pH}$ is $7.0-7.5$, turbidity is $15-25 \mathrm{NTU}$, $\mathrm{UV}_{254}$ is $0.031-0.084 \mathrm{~cm}^{-1}$

\subsection{Test instruments and reagents}

Instruments: 2100P turbidimeter, HACH company; WGZ200 colorimeter, Shanghai Xinrui Instrument Co., Ltd.; T6 new century UV spectrophotometer, Beijing Puxi General Instrument Co., Ltd.; MY3000-6F intelligent coagulation test mixer, Wuhan Meiyu Instrument Co.,Ltd.; portable $\mathrm{pH}$ meter, Taizhou Zhengda science and education equipment factory; FK-H3 digital display constant temperature magnetic stirrer, Fangke instrument (Changzhou) Co., Ltd.; FA2204N electronic balance, Shanghai Qinghai Instrument Co., Ltd.

Reagents: polymerized ferric sulfate (PFS), iron content $18.5 \%$, basicity 9.0-14, Pb 2.0-3.0, arsenic 0.0008, lead 0.0015 , insoluble matter 0.5 , Tianjin dingshengxin Chemical Co., Ltd.; poly (PAC), $\mathrm{AL}_{2} \mathrm{O}_{3}$ solid content $30 \%$, $\mathrm{pH}$ (1\% aqueous solution)3.5-5.0, Gongyi Zhongbang water purification material Co.,Ltd.; other reagents were analytical pure.

\subsection{Experimental methods}

The influence of PAC and PFS coagulant on turbidity, Chroma and $\mathrm{UV}_{254}$ in Pi River water under different

*Corresponding author E-mail:944605217@qq.com 
mixing ratio, dosage, turbidity of raw water was investigated by coagulation beaker method, and the

Table1. coagulation sedimentation test procedure

\begin{tabular}{llll} 
& Table1. & coagulation sedimentation test procedure \\
\hline Segment number & speed/(r.min $\left.{ }^{-1}\right)$ & Time $/ \mathrm{s}$ & dosing \\
\hline 1 & 300 & 20 & dosing \\
2 & 175 & 300 & No dosing \\
3 & 55 & 600 & No dosing \\
4 & 0 & 1800 & No dosing \\
\hline
\end{tabular}

\subsection{Test items and analysis methods}

\subsubsection{Determination of turbidity, color, $U V_{254^{*}}, \mathrm{pH}$}

The turbidity was measured by $2100 \mathrm{P}$ portable turbidimeter.The chromaticity was measured by WGE200 desktop colorimeter. $U_{254}$ and $\mathrm{UV}_{365}$ were measured by $\mathrm{T} 6$ new century ultraviolet spectrophotometer, $\mathrm{UV}_{254^{*}=}$ $\mathrm{UV}_{254}-\mathrm{UV}_{365}$, and the $\mathrm{pH}$ value was measured by portable $\mathrm{pH}$.

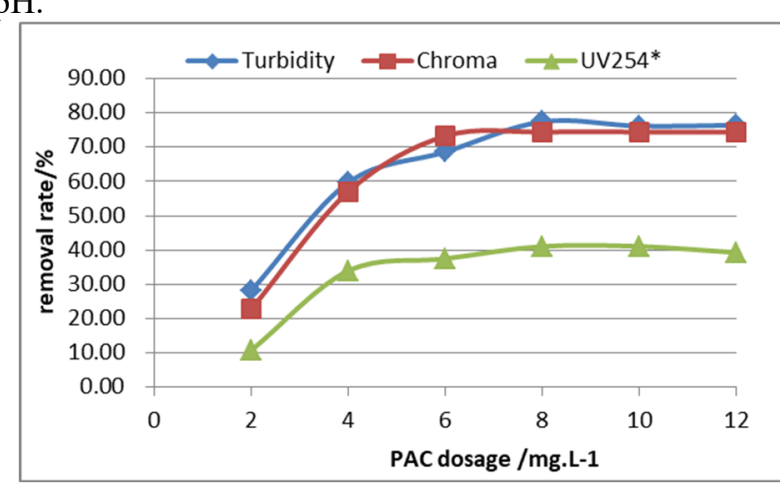

\section{Results and discussion}

\subsection{Effect of PFS and PAC dosage on turbidity reduction, decoloration and organic matter removal}

The removal effects of different PFS and PAC dosage on turbidity, Chroma and $\mathrm{UV}_{254 *}$ in Pi River water are shown in Fig.1 and Fig.2.

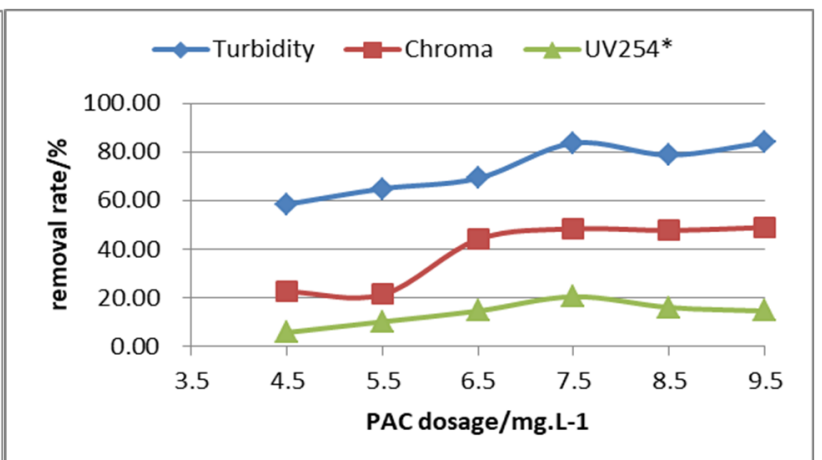

Fig.1 Effect of PAC dosage on flocculation

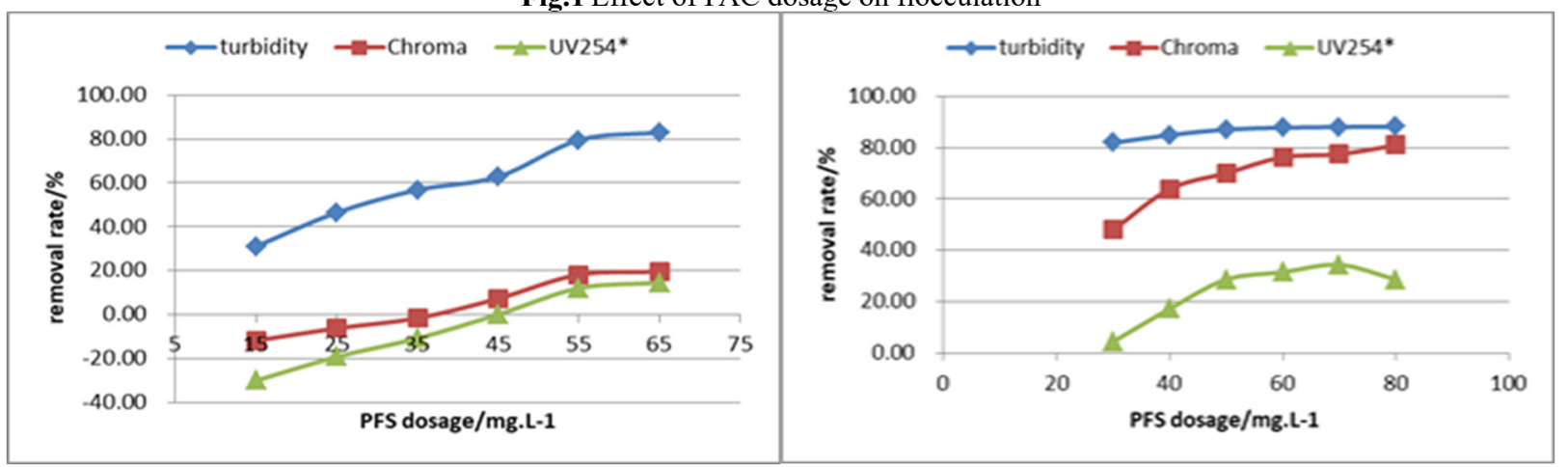

Fig.2 Effect of PFS dosage on flocculation

It can be seen from Figure 1 that with the increase of PAC dosage, the removal rate of turbidity, Chroma and $\mathrm{UV}_{254 *}$ show a similar trend. When the dosage of PAC increases from $2 \mathrm{mg} / \mathrm{L}$ to $12 \mathrm{mg} / \mathrm{L}$, the turbidity removal rate first increases from $28.07 \%$ to $77.43 \%$ and then slow decreases to $76 \%$, the color removal rate increases from $22.67 \%$ to $74.42 \%$, the $\mathrm{UV}_{254} *$ removal rate first increases from $10.71 \%$ to $41.07 \%$, and then slow decreases to $39 \%$, The main reasons are as follows: when the dosage is too small, the colloid in the water has flocculation reaction, the colloid is small and less suspended in the water, so the flocculation effect is poor; With the increase of flocculant dosage, PFS is a high polymer flocculant, which has the functions of adsorption bridging, electric neutralization, etc., making colloidal ions in water destabilize and finally precipitate; When too much flocculant is added, the negatively charged particles in water will change, and the colloidal particles will repel each other, which shows that the turbidity removal effect will decrease. In order to find out the best dosage of PAC, the dosage from $4.5 \mathrm{mg} / \mathrm{L}$ to $9.5 \mathrm{mg} / \mathrm{L}$ was selected, When the dosage of PAC is $7.5 \mathrm{mg} / 1$, the coagulation effect is the best, and the removal rates of turbidity, Chroma and $\mathrm{UV}_{254 *}$ are $83.75 \%, 48.47 \%$ and $20.59 \%$ respectively.

It can be seen from Figure 2 that with the increase of PFS dosage, the removal rate of turbidity, Chroma and $\mathrm{UV}_{254 *}$ show a similar trend too. When the dosage of PFS increases from $30 \mathrm{mg} / \mathrm{L}$ to $80 \mathrm{mg} / \mathrm{L}$, the turbidity removal rate increases from $81.88 \%$ to $88.19 \%$, the color removal rate increases from $47.86 \%$ to $80.93 \%$, and the $\mathrm{UV}_{254}$ removal rate increases from $4.29 \%$ to $34.29 \%$, Although the removal rate of turbidity and Chroma 
continues to increase, and the removal rate of $\mathrm{UV}_{254 *}$ increases slowly, the $\mathrm{pH}$ value in raw water drops rapidly, from the initial 7.4 to below 5. Considering that the $\mathrm{pH}$ of the factory water is greater than 6.5 , the optimal dosage of PFS is determined to be $35 \mathrm{mg} / 1$.In conclusion, when the dosage of PFS is $35 \mathrm{mg} / \mathrm{L}$, the dosage of PAC is $7.5 \mathrm{mg} /$ $\mathrm{L}$ the flocculation effect is the best.

Table2. Influence of raw water turbidity on

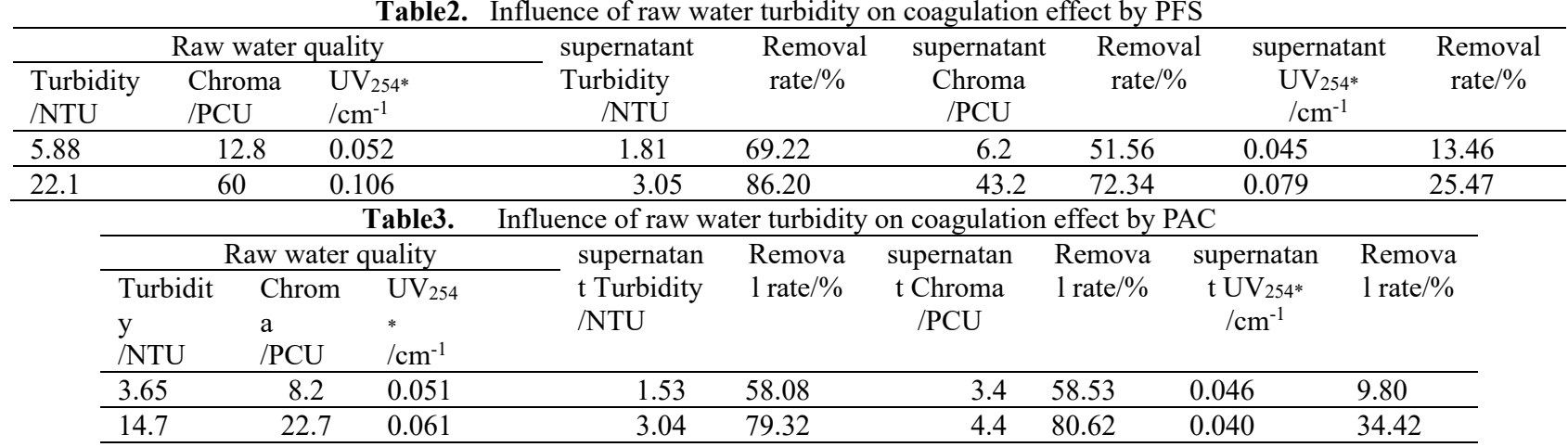

When the turbidity of raw water is higher than 10NTU, the removal efficiency of turbidity, Chroma and $\mathrm{UV}_{254 *}$ is better than that of raw water less than 10NTU.When PFS is added, the removal rates of turbidity, Chroma and $\mathrm{UV}_{254}$ increase by $17 \%, 20 \%$ and $12 \%$ respectively. When PAC is added, the removal rates of turbidity, Chroma and $\mathrm{UV}_{254 *}$ increase by $20 \%, 22 \%$ and $25 \%$ respectively. The reason is with the increase of initial turbidity of water sample, on the one hand, the number of suspended particles in water sample increases, the probability of particles colliding with each other after destabilization in the coagulation process becomes larger, the rate of getting flocs after more suspended particles aggregate is faster, and the flocs have larger specific surface area, which can increase the removal rate of $\mathrm{UV}_{254 *}$ and turbidity through the synergistic effect of adsorption and co sedimentation; on the other hand, the positively charged poly nuclear complexes formed by the hydrolysis of PAC and PFS will compress the electric double layer and reduce the energy consumption $\zeta,{ }^{[4]}$ the adsorption or sweeping ability of flocs enhanced the removal efficiency of $\mathrm{UV}_{254} *$ and turbidity.

\subsection{PAC and PFS were added separately and mixed according to different proportions}

The dosage of PAC was $7.5 \mathrm{mg} / 1$, and the dosage of PFS was 1, 3, 5, 7 and 9 times of PAC dosage, respectively. The results are shown in Figure 3

\subsection{Influence of raw water turbidity on removal efficiency}

The raw water is made of Pi River water and tap water in a certain proportion. The dosage of PFS is $35 \mathrm{mg} / \mathrm{L}$. The dosage of PAC is $7.5 \mathrm{mg} / \mathrm{L}$. The experimental results are shown in Table 2 and Table 3. 
dosage is at least 3 times of PAC dosage, but even if PFS dosage is increased It is also difficult to achieve PAC removal effect in terms of quantity, Chroma and UV254* removal effect, and the $\mathrm{pH}$ in the water drops rapidly from 7.1 to 6.1. Therefore, the dosage of PFS should be strictly controlled to ensure that the $\mathrm{pH}$ of the effluent water meets the requirements.

In order to further study the removal effect of PAC and PFS, the mixed dosage of PAC and PFS was $7.5 \mathrm{mg} / 1$ The mass ratio of PAC to PFS was 1:1, 2:1, 3:1, 4:1, 5:1, 6:1. It is found from Fig. 4 that with the increase of PAC content in the composite coagulant, the removal efficiency of turbidity and chromaticity is improved, increasing by $10 \%$ and $12 \%$ respectively. However, UV254* does not change much, and even decreases.

\section{Conclusion}

(1) Both PAC and PFS can reduce the turbidity, chromaticity and UV254* in Pi River water. The $\mathrm{pH}$ of the water does not decrease after PAC coagulation, but PFS coagulation can significantly affect the $\mathrm{pH}$ of raw water. Therefore, if PFS coagulation is selected, the dosage must be strictly controlled, and the removal efficiency of PAC coagulation is higher than that of PFS coagulation.

(2) In the low turbidity Pi River water, the turbidity of raw water, the separate dosage of two coagulants, and the mixed dosage according to different proportions will have an impact on the coagulation. When PAC dosage is $7.5 \mathrm{mg} / \mathrm{l}$, PFS dosage is $35 \mathrm{mg} / \mathrm{L}$, initial turbidity of water sample is less than 20NTU, $\mathrm{pH}$ value is neutral. Under the optimum conditions, the removal rates of UV254* by PAC were 9.8\%-34.42\%, turbidity 58.08-79.32 and Chroma $58.53-80.62$; the removal rates of UV254* by PFS were 13.46\%-25.47\%, turbidity $69.22-86.20$ and Chroma 51.56-72.34. the mass ratio of PAC to PFS is $1: 1$ to $6: 1$, the removal efficiency of turbidity and Chroma increased by about $10 \%$, but the removal efficiency of UV254* did not change or even decreased.

(3) Although PFS is better than PAC in turbidity removal, PAC is more suitable for the removal of turbidity, Chroma and UV254* in Pihe River considering the obvious decrease of $\mathrm{pH}$ after PFS dosing.

\section{Acknowledgement}

his research was funded by university natural science research project in Anhui .No.KJ2019A0621.

\section{References}

1. Zhang C.Some thoughts on water quality safety of Pi River main canal.Water conservancy science and technology and economy,2014,20(10):15-16.

2. Zhang X.S. Research on Synthesis and Phosphorus Removal Effect of Composite Flocculant PFSPDMDAAC. Guangdong chemical engineering,2017,44(14):55-56.

3. Wang Z.Y, Dai H.L, Zhou Z, et al.Research and Application of Low Temperature and Low Turbidity
Water Treatment Technology. TECHNOLOGY OF WATER TREATMENT, 2016,42(10):20-23.

4. BIRDI K S. Surface and Colloid Chemistry: Principles and Applications.1 st edition.CRC Press, 2010. 